\title{
Transplantation of bone marrow-derived endothelial progenitor cells attenuates myocardial interstitial fibrosis and cardiac dysfunction in streptozotocin-induced diabetic rats
}

\author{
YONGXIA CHENG ${ }^{1}$, SUFEN GUO ${ }^{1}$, GUIBO LIU ${ }^{2}$, YUKUAN FENG $^{2}$, BIN YAN $^{1}$, \\ JIANBO YU ${ }^{1}$, KEJIAN FENG ${ }^{2}$ and ZHIQIANG LI ${ }^{1}$ \\ Departments of ${ }^{1}$ Pathology and ${ }^{2}$ Anatomy, Mudanjiang Medical College, Mudanjiang, \\ Heilongjiang 157011, P.R. China
}

Received April 26, 2012; Accepted June 29, 2012

DOI: $10.3892 /$ ijmm.2012.1083

\begin{abstract}
Diabetic cardiomyopathy (DCM) is a progressive disease of the heart muscle and the third most common cause of heart failure. In the present study, we evaluated the effects of bone marrow-derived endothelial progenitor cell (EPC) transplantation on the development of DCM in a streptozotocin (STZ)-induced diabetic rat model. Ex vivo generated, characterized and cultivated rat EPCs were identified by flow cytometry of their surface markers. EPCs were transplanted intravenously into rats through the tail vein 6 weeks after they were challenged with STZ and the rats were sacrificed 4 weeks later. Before sacrifice, left ventricular (LV) catheterization was performed to evaluate the cardiac function. Myocardium sections were stained with Masson's trichrome staining to investigate myocardial collagen contents. Fibrosis-, apoptosis- and oxidative stress-related gene expressions were analyzed by western blot analysis. Transplantation of EPCs alleviated the impaired cardiac function associated with diabetes and decreased the collagen volume in diabetic myocardium resulting in improved cardiac function. Furthermore, EPC transplantation decreased the expression of type I collagen, Bax, caspase-3 and p67phox, while increasing the expression of Bcl-2 and manganese superoxide dismutase (MnSOD). Taken together, our results suggest that transplantation of EPCs improved cardiac function in the rat DCM
\end{abstract}

Correspondence to: Dr Zhiqiang Li, Department of Pathology, Mudanjiang Medical College, 3 Tongxiang Street, Mudanjiang, Heilongjiang 157011, P.R. China

E-mail: mdjlizhiqiang@hotmail.com

Dr Kejian Feng, Department of Anatomy, Mudanjiang Medical College, 3 Tongxiang Street, Mudanjiang, Heilongjiang 157011, P.R. China

E-mail: kejian.feng@hotmail.com

Key words: endothelial progenitor cell, diabetic cardiomyopathy, apoptosis, myocardial fibrosis, oxidative stress model, likely through inhibition of cardiomyocyte apoptosis and attenuating myocardial fibrosis.

\section{Introduction}

Diabetic cardiomyopathy (DCM), which occurs in type I and II diabetes, is a substantial risk factor for the subsequent development of heart failure and increased mortality (1). Its pathological substrate is characterized by early-onset diastolic dysfunction and late-onset systolic dysfunction in the absence of coronary artery disease, hypertension or significant valvular heart disease $(2,3)$. The pathological mechanism of DCM may be due to metabolic disturbances, reactive hypertrophy, small vessel disease, autonomic dysfunction, insulin resistance and myocardial fibrosis. At present, conventional therapies for DCM include glycemic control and early administration of neurohormonal antagonists. DCM, however, has yet to be completely prevented $(4,5)$. Therefore, the development of more effective therapeutic strategies for DCM is necessary.

Endothelial progenitor cells (EPCs) are a subset of bone marrow-derived stem cells present in peripheral circulation that have the potential to differentiate into functional and mature endothelial cells. Since their revolutionary discovery by Asahara et al (6) in 1997, EPCs have been demonstrated to be of importance in postnatal vasculogenesis through pivotal bioactivities, mobilization, homing, migration, differentiation and proliferation in angiovasculogenic tissues (7). However, decreased levels of circulating EPCs have been reported in a wide range of pathological conditions, such as chronic kidney disease (8), hypercholesterolemia (9), rheumatoid arthritis (10), obesity (11) and coronary artery disease (12). Furthermore, an altered status of circulating EPCs represents a diagnostic biomarker for endothelial dysfunction and individual diseases. Due to their role in enhacing angiogenesis, there has been a growing interest in using EPCs as therapeutic agents to supply the potent origin of neovascularization under pathological conditions (13-16). Endothelial dysfunction is a hallmark of diabetic vascular complications and reduction in circulating EPCs and functional impairment of cultured EPCs have been reported in type I and II diabetic patients $(17,18)$. 
Recently, the ex vivo therapy of culture-expanded EPC transplantation has been proposed as a novel therapeutic strategy for diabetes mellitus and its associated vascular complications (19-21). However, the possibility of cellular therapy with EPCs for the treatment of DCM has yet to be investigated.

Based on recent advances in studies of EPCs in diabetes and the lack of treatment strategies that improve cardiac repair in diabetics, we hypothesized that EPCs might improve the cardiac function of diabetic rats. To test this hypothesis, a streptozotocin (STZ)-induced diabetic rat model was used. Subsequent to EPC isolation and proliferation in vitro, a series of experiments was conducted to determine the changes in morphology and other biological characteristics, and to assess whether transplantation of EPCs is to be considered a therapeutic candidate for the treatment of DCM.

\section{Materials and methods}

Animals and ethics statement. Male Sprague-Dawley rats at a postnatal age of 7-8 weeks (body weight, 200-220 g) were obtained from the Experimental Animal Center of the Mudanjiang Medical College. The rats were kept on straw bedding in cages under light/dark cycle conditions and were acclimatized to the laboratory conditions for 7 days prior to commencing the experiments. The animals were fed standard rodent diet and water ad libitum, unless otherwise indicated. The experiment protocol was approved by the Institutional Animal Care and Use Committee and was performed in accordance with the Guide for the Care and Use of Laboratory Animals (NIH Publication no. 85-23, National Academy Press, Washington, DC, revised 1996).

Isolation and culture of putative EPCs. EPCs were isolated from the femurs and tibias of donor rats, according to the method reported in previous studies $(22,23)$. In brief, the animals were anesthetized with chloral hydrate $(300 \mathrm{mg} / \mathrm{kg})$ and sacrificed using cervical dislocation. Femur and tibias were excised from each rat under sterile conditions, while their bone marrow cavities were rinsed with phosphatebuffered saline (PBS). The rinsing solution was then subjected to density gradient centrifugation with Histopaque-1083 (Sigma, St. Louis, MO, USA). The obtained mononuclear cells were grown in M199 medium supplemented with $20 \%$ fetal bovine serum (FBS), $0.05 \mathrm{mg} / \mathrm{ml}$ bovine pituitary extract (Invitrogen Life Technologies, Carlsbad, CA, USA), antibiotics and $10 \mathrm{U} / \mathrm{ml}$ heparin on fibronectin-coated dishes. Seeded cells were cultured in a humidified incubator at $37^{\circ} \mathrm{C}$ in $5 \% \mathrm{CO}_{2}$. After 10 days of culture, the cells were detached with trypsin-EDTA and passed through a cell strainer. To detect transplanted EPCs, cells were pre-labelled with a red fluorescent marker, CM-Dil (Invitrogen Life Technologies) following the manufacturer's instructions.

Flow cytometry. To confirm that the isolated EPCs maintain their phenotypic characteristics after culture, flow cytometry was performed on freshly isolated cells (at day 0), as well as after 10 days of culture. Briefly, after being washed with PBS, cells were incubated for $30 \mathrm{~min}$ at $4^{\circ} \mathrm{C}$ with mouse anti-rat antibodies for CD31 and CD34 (Santa Cruz Biotechnology, Inc., Santa Cruz, CA, USA) and rabbit anti-rat antibodies for CD133 and vascular endothelial growth factor receptor 2 (VEGFR2) (Abnova, Taipei, Taiwan), followed by anti-mouse or anti-rabbit fluorescein isothiocyanate (FITC)-labeled secondary antibodies. Finally, flow cytometry was performed with a FACSCalibur cytometer (BD Biosciences, San Jose, CA, USA) and data were analyzed using CellQuest software.

Induction of diabetes. A rat DCM model was induced as described previously (24). In brief, 30 rats were injected intraperitoneally with $60 \mathrm{mg} / \mathrm{kg}$ body weight of STZ (Sigma) prepared in $0.1 \mathrm{ml}$ citrate buffer $(0.1 \mathrm{mmol} / 1, \mathrm{pH} 4.5)$ to induce diabetic conditions, while 8 rats received an equal volume of vehicle and served as a non-diabetic group. Seven days subsequent to STZ injection, blood samples were collected from the tail vein after $12 \mathrm{~h}$ of fasting. Nineteen rats reached the diabetic rat standard of a fasting blood glucose level $>300 \mathrm{mg} / \mathrm{dl}$, while the normal value in the control rats injected with vehicle ranged from 80 to $120 \mathrm{mg} / \mathrm{dl}$. Eight weeks subsequent to vehicle and STZ injection, 16 diabetic rats remained, while the control group survived. The remaining animals were used for additional experiments.

EPC transplantation. Sixteen diabetic rats were randomized into the EPC and DCM groups ( $n=8$ per group). Rats in the EPC group were transplanted intravenously through the tail vein $\sim 1 \times 10^{6}$ EPCs (in $0.5 \mathrm{ml}$ culture medium). Rats in the control and DCM groups received an equal volume of culture medium at the same time point. The rats were sacrificed at 4 weeks subsequent to EPC transplantation. Prior to being sacrificed, blood was drawn to detect the level of fasting blood glucose.

Echocardiographic evaluation. Prior to being sacrificed, each rat was anesthetized with chloral hydrate $(300 \mathrm{mg} / \mathrm{kg})$. A catheter (PE-50; Becton-Dickinson, Parsippany, NJ, USA) was inserted into the right carotid artery and then advanced into the left ventricular (LV) chamber to measure the following indices: heart rate (HR), LV systolic blood pressure (LVSP), $\mathrm{LV}$ end diastolic blood pressure (LVEDP), maximum rising rate of $\mathrm{LV}$ pressure $(\mathrm{LV}+\mathrm{dp} / \mathrm{dtmax})$ and minimum declining rate of LV pressure (LV-dp/dtmin), as delineated previously (25). The rectal temperature was maintained at $36-38^{\circ} \mathrm{C}$ using a heating pad throughout the procedure. Rats were sacrificed immediately after echocardiographic evaluation. The hearts were harvested and cut into 2 sections, 1 was immersed in $10 \%$ formalin solution for Masson's trichrome staining, while the other in liquid nitrogen for western blot analysis.

Masson's trichrome staining. The middle of the LV was fixed in $10 \%$ neutral-buffered formalin overnight, embedded in paraffin and sectioned to a thickness of $5 \mu \mathrm{m}$. The sections were stained with Masson's trichrome staining to measure interstitial fibrosis. Interstitial collagen was quantified at a final magnification of $\mathrm{x} 200$ with a microscope (BA400 Binocular Microscope; Motic, Xiamen, China) connected to a video camera. The resulting image was processed on a computer image-analysis system. The content of interstitial collagen (expressed as the fractional area of the entire cross section) was averaged on 9 fields selected across the wall thickness in the septum and the free wall. 
Protein extraction and western blot analysis. Western blot analysis was performed as previously described (24). The hearts were lysed in radioimmunoprecipitation (RIPA) assay buffer (Beyotime Institute of Biotechnology, Haimen, China). The heart tissue homogenates were clarified by centrifugation and protein concentrations of the lysates were determined using a bovine serum albumin standard line. Equal amounts of protein were boiled at $100^{\circ} \mathrm{C}$ for $10 \mathrm{~min}$ and chilled on ice, subjected to sodium dodecyl sulfate polyacrylamide gel electrophoresis (SDS-PAGE) analysis, and then electrotransferred to polyvinylidene fluoride (PVDF) membranes (Millipore, Bedford, MA, USA). The membranes were blocked with 5\% non-fat dry milk (w/v) and then incubated overnight at $4^{\circ} \mathrm{C}$ with rabbit anti-caspase-3, rabbit anti-Bcl-2, mouse anti-Bax, rabbit anti-collagen I, rabbit anti-manganese superoxide dismutase (MnSOD) and rabbit anti-p67phox (dilution 1:1000; Abcam, Cambridge, MA, USA), followed by their respective horseradish peroxidase-conjugated secondary antibodies. After extensive washing, the band detection was revealed by an ECL plus chemiluminescence kit (Millipore).

Statistical analysis. Data are presented as the means \pm standard deviation (SD). Statistical differences in the groups were evaluated by one-way analysis of variance, while the least-significant difference (LSD) test was used for multiple comparisons. Graphs were plotted and statistical calculations were performed using SigmaPlot 12.0 (Systat Software, Inc., San Jose, CA, USA). P $<0.05$ was considered to indicate statistically significant differences.

\section{Results}

Characterization of bone marrow-derived EPCs. After a 10-day culture, the isolated cells were observed as bone marrow-derived EPC colony-forming units, exhibiting endothelium-specific cord-like structures. Flow cytometry showed that endothelial lineage-specific markers such as CD31, CD34, CD133 and VEGFR2 significantly increased in 10 days subsequent to plating (Fig. 1). The expression changes in these factors are characteristic of those of EPCs (26).

In vivo tracking of EPCs in myocardial tissues. The trafficking of EPCs into the myocardial tissues of STZ-induced diabetic rats was monitored using double staining of CM-Dil and 4',6-diamidino-2-phenylindole (DAPI). As shown in Fig. 2, numerous CM-Dil-labeled EPCs showing red fluorescence adoptively transferred into the myocardial tissues of STZ-induced diabetic rats, indicating that EPCs had the potential to migrate into the myocardium in the STZ-induced diabetic model at 4 weeks after transplantation.

EPC transplantation improved myocardial function in diabetic rats. Twelve weeks after the induction of diabetes, rat cardiac function was evaluated by invasive hemodynamic measurements. The metabolic and hemodynamic parameters of the animals are presented in Table I. A lower LVSP and higher LVEDP were observed in the DCM compared to the control group. STZ-diabetic animals showed significant impairments in the contraction $(+\mathrm{dp} / \mathrm{dt})$ and relaxation rate (-dp/dt), compared to the control animals, indicating that the
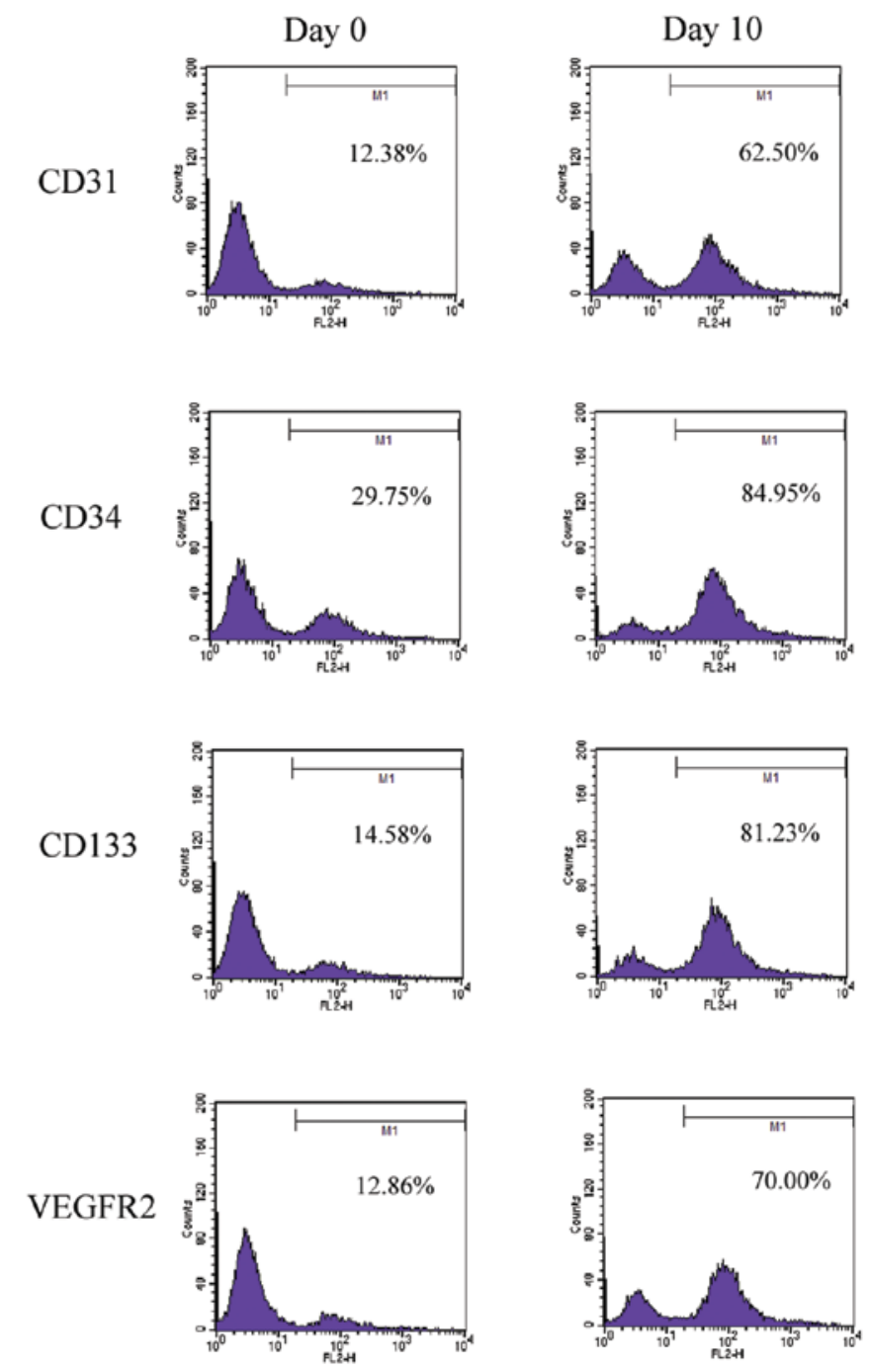

Figure 1. Flow cytometry of the isolated rat EPCs at day 0 and 10 days subsequent to culture. Values in the graph show the percentage of each type of marker-positive cells. Plot is representative of at least 3 independent experiments.

systolic and diastolic functions in the diabetic rat heart were significantly impaired. Four weeks subsequent to EPC transplantation, the above-mentioned hemodynamic abnormalities were notably attenuated. However, there were no significant differences in the heart rate in the 3 groups. In addition, plasma glucose concentrations in diabetic rats exceeded $16.7 \mathrm{mmol} / \mathrm{l}$, however, these concentrations decreased significantly subsequent to EPC transplantation.

\section{EPC transplantation attenuated myocardial interstitial} fibrosis in diabetic rats. Myocardial fibrosis is a pathological hallmark in the development of DCM. Masson's trichrome staining was used to examine whether EPC transplantation affected the myocardial fibrosis in a STZ-induced diabetic rat model. Obvious interstitial and perivascular fibrosis were detected in the myocardium of the DCM group compared with the control group (Fig. 3A-C). Quantification of the fibrosis area showed a statistically significant increase of fibrosis in the DCM group, whereas EPC transplantation markedly attenuated fibrosis progression in the diabetic hearts $(\mathrm{P}<0.01)$ (Fig. 3D). Moreover, western blot analysis showed 
Table I. Hemodynamic parameters evaluated by invasive measurements.

\begin{tabular}{lccc}
\hline & \multicolumn{3}{c}{ Groups } \\
\cline { 2 - 4 } Parameters & Control (n=8) & DCM (n=8) & EPC (n=8) \\
\hline Plasma glucose (mmol/l) & $4.7 \pm 0.8$ & $28.4 \pm 2.0^{\mathrm{a}}$ & $13.7 \pm 2.2^{\mathrm{b}}$ \\
HR (beats/min) & $327 \pm 20$ & $338 \pm 32$ & $307 \pm 22$ \\
LVSP (mmHg) & $123.4 \pm 7.9$ & $95.6 \pm 7.1^{\mathrm{a}}$ & $109.5 \pm 5.6^{\mathrm{b}}$ \\
LVEDP (mmHg) & $4.7 \pm 0.8$ & $15.4 \pm 2.3^{\mathrm{a}}$ & $9.2 \pm 2.2^{\mathrm{b}}$ \\
+dp/dt (mmHg/sec) & $5697 \pm 304$ & $2761 \pm 458^{\mathrm{a}}$ & $3762 \pm 473^{\mathrm{b}}$ \\
-dp/dt (mmHg/sec) & $4788 \pm 337$ & $2614 \pm 281^{\mathrm{a}}$ & $3524 \pm 315^{\mathrm{b}}$ \\
\hline
\end{tabular}

Values are presented as the mean $\pm \mathrm{SD}$; HR, heart rate; LVSP, left ventricle systolic pressure; LVEDP, left ventricle end diastolic pressure; $+\mathrm{dp} / \mathrm{dt}$, maximum rate of left ventricle pressure increase; -dp/dt, maximum rate of left ventricle pressure decrease; ${ }^{\text {a }}<0.01 \mathrm{vs}$. the control group; ${ }^{\mathrm{P}}<0.01$ vs. the $\mathrm{DCM}$ group.
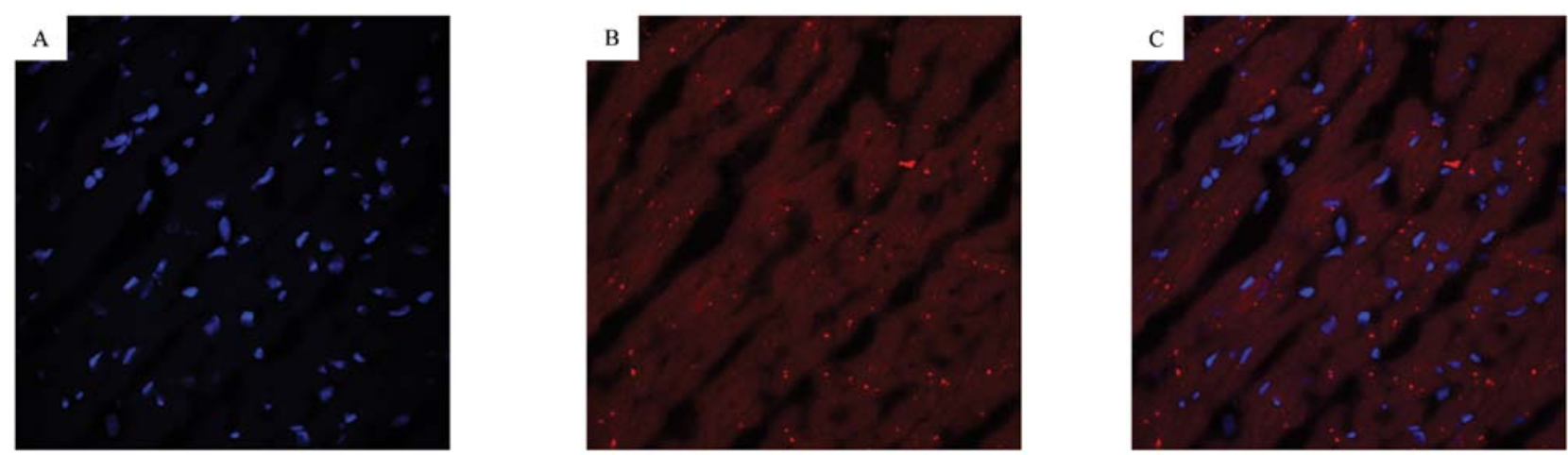

Figure 2. Identification of CM-Dil-stained EPCs (red fluorescence) in the cardiac tissues of rats from the EPC group. (A) CM-Dil staining; (B) DAPI staining; (C) merged results. Original magnification, $\mathrm{x} 400$.
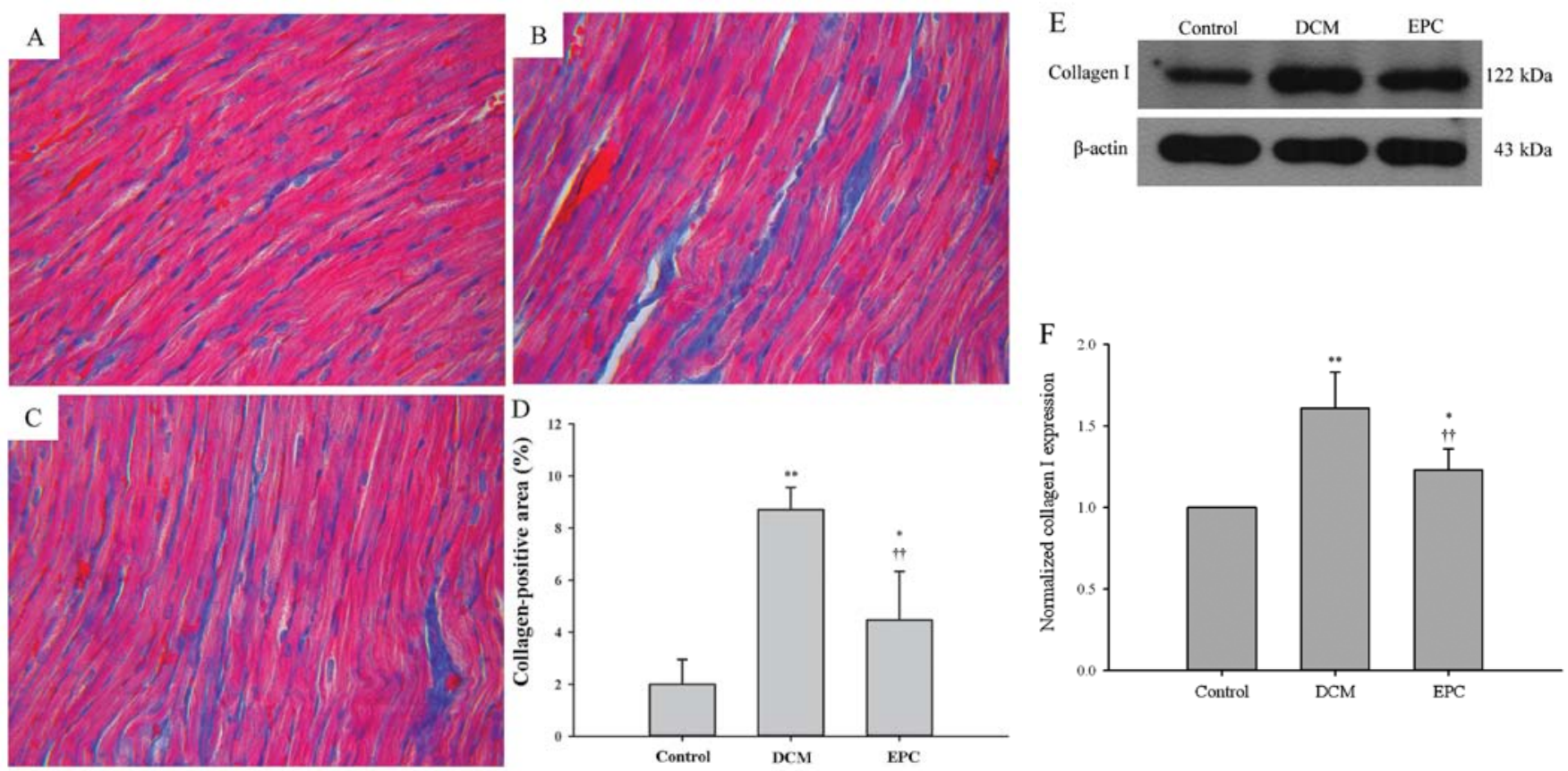

Figure 3. Effects of EPC transplantation on myocardial interstitial fibrosis in DCM. Representative images showing Masson's trichrome staining of the myocardium from the (A) control, (B) DCM and (C) EPC groups. Areas stained blue show the fibrotic infiltration. (D) Quantitative analysis of fibrosis. Values are presented as the mean $\pm \mathrm{SD}(\mathrm{n}=8) ;{ }^{*} \mathrm{P}<0.05,{ }^{* *} \mathrm{P}<0.01$ vs. the control group; ${ }^{\dagger} \mathrm{P}<0.01$ vs. the DCM group. (E) Western blot analysis of type I collagen expression in the myocardium of STZ-induced diabetic rats. Representative blots are shown and the protein size is expressed in $\mathrm{kDa}$. (F) Densitometric quantification data are expressed as the intensity ratio of the target genes to $\beta$-actin (mean $\pm \mathrm{SD}, \mathrm{n}=8$ ). ${ }^{*} \mathrm{P}<0.05,{ }^{* *} \mathrm{P}<0.01$ vs. the control group; ${ }^{\dagger} \mathrm{P}<0.01$ vs. the $\mathrm{DCM}$ group. 
A
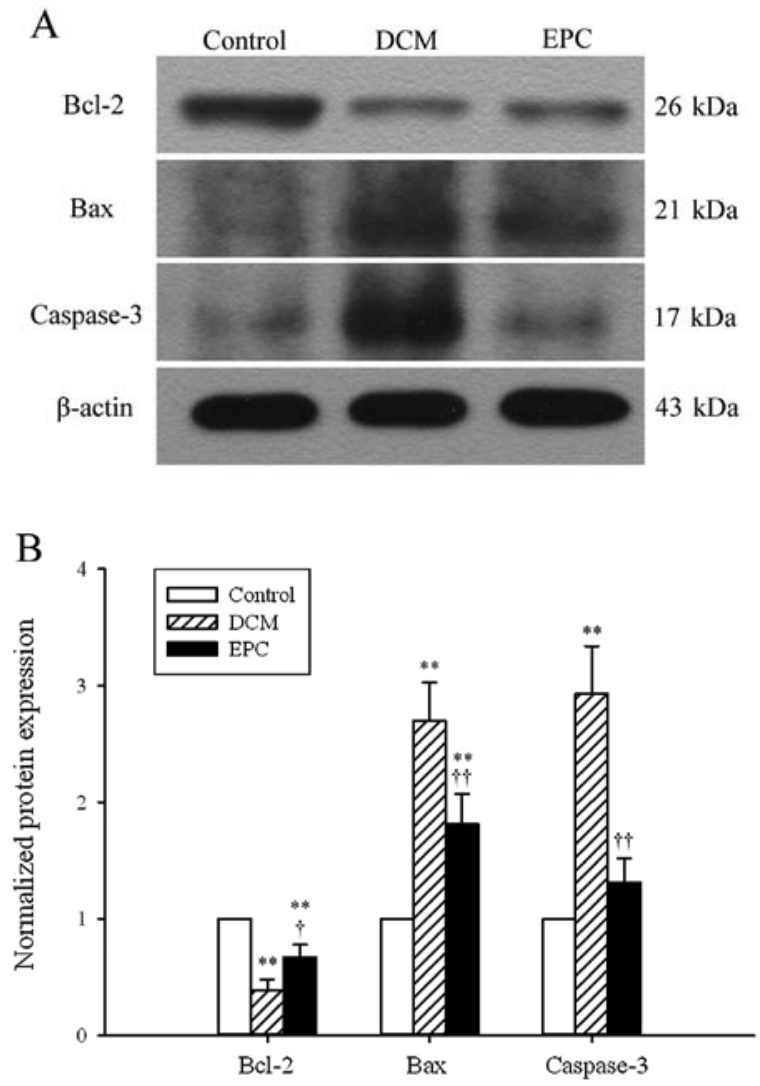

Figure 4. Western blot analysis of apoptosis-related genes in the myocardium of STZ-induced diabetic rats. (A) Blots shown are representative of 3 independent experiments. (B) Quantification of ILK protein from the 3 independent experiments, optimized to $\beta$-actin $(n=8) .{ }^{* *} \mathrm{P}<0.01$ vs. the control group; ${ }^{\dagger} \mathrm{P}<0.05,{ }^{\dagger} \mathrm{P}<0.01$ vs the $\mathrm{DCM}$ group.

that the type I collagen expression was markedly upregulated in the DCM group, but inhibited by the EPC transplantation $(\mathrm{P}<0.01)$ (Fig. 3E-F). These results suggest that EPC transplantation has the potential to attenuate diabetes-induced myocardial interstitial fibrosis.

EPC transplantation protected cardiomyocytes against apoptosis in diabetic rats. Cardiomyocyte apoptosis is a critical process in the pathogenesis of DCM. The expression of apoptotic markers, such as Bcl-2, Bax and caspase-3, was examined by western blot analysis in order to investigate whether or not transplantation of EPCs improved myocardial function by protecting cardiomyocytes against apoptosis. Bcl-2 was found to be downregulated, whereas Bax and caspase-3 were upregulated in the DCM group (Fig. 4). These changes, however, were markedly attenuated by the administration of EPCs. Since Bcl-2 is characterized by anti-apoptosis, while Bax and caspase- 3 by pro-apoptosis, our results suggest that transplantation of EPCs has the potential to protect cardiomyocytes against apoptosis.

EPC transplantation prevented oxidative stress in diabetic rats. The effects of EPC transplantation on the endogenous pro-oxidants and antioxidants, including p67phox and MnSOD, were determined by western blot analysis. The results showed an elevated expression of p67phox in dietetic hearts, suggesting that an endogenous pro-oxidant system
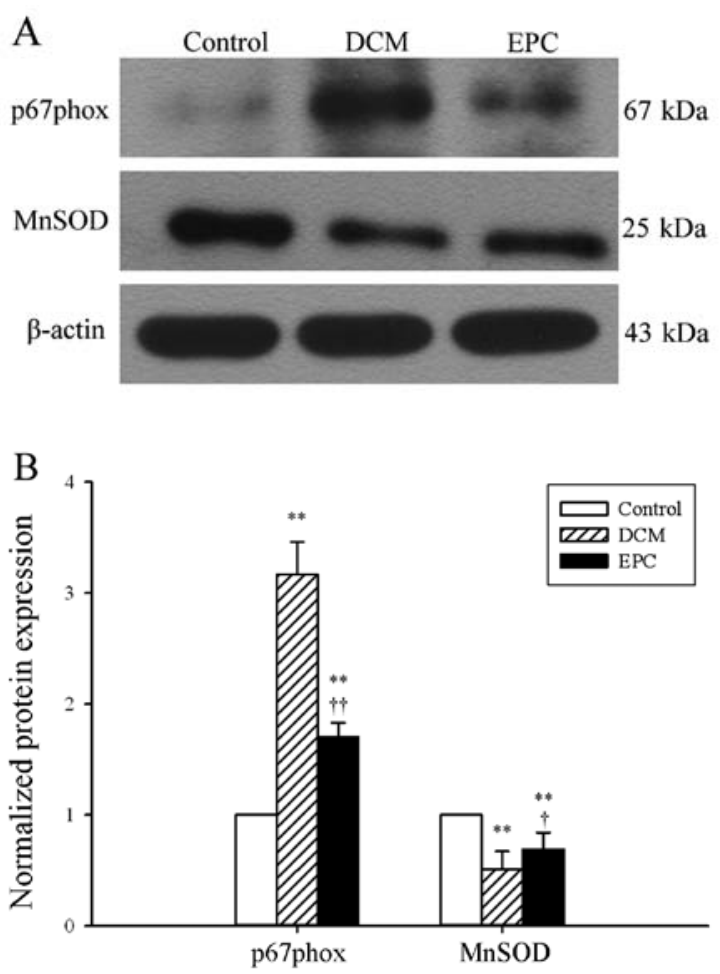

Figure 5. Protein expressions of p67phox and MnSOD in the myocardium of STZ-induced diabetic rats were determined by western blot analysis (A) Representative blots are shown and protein size is expressed in kDa. (B) Quantitative data of protein levels were expressed as the intensity ratio of target proteins to $\beta$-actin. Data present the mean $\pm \mathrm{SD}(\mathrm{n}=8) .{ }^{* *} \mathrm{P}<0.01$ vs the control group; ${ }^{\dagger} \mathrm{P}<0.05,{ }^{\dagger} \mathrm{P}<0.01$ vs. the $\mathrm{DCM}$ group.

was triggered under diabetic conditions, which may further aggravate oxidative stress. EPC transplantation markedly attenuated the p67phox expression (Fig. 5). However, a statistically significant decrease in the MnSOD expression in STZ-induced diabetic hearts was observed, compared to control hearts. Transplantation of EPCs resulted in a statistically significant increase in the MnSOD expression in the DCM group. These findings demonstrate that transplantation of EPC exerts its protective effects by targeting the nicotinamide adenine dinucleotide phosphate (NADPH) oxidase and mitochondrial redox enzymes.

\section{Discussion}

In this study, we have shown that transplantation of EPCs significantly improved cardiac function and attenuated cardiac fibrosis in a rat model of STZ-induced diabetes, as evidenced by echocardiography, Masson's trichrome staining and the analysis of type I collagen expression. Moreover, EPC implantation effectively prevented STZ-induced cardiac apoptosis and oxidative stress, indicating the possible mechanisms whereby EPC transplantation exerts its cardioprotective effects against myocardial fibrosis and improves diastolic dysfunction in diabetic rats. The present study is known to be the first to report the potential therapeutic effects of EPC transplantation in an animal model of DCM.

Although in recent years an excessive number of studies have been available on EPCs, the definition of EPCs has yet to be determined due to the lack of unique cell surface 
molecules that specifically recognize EPCs $(27,28)$. Currently, the most widely accepted definition is the co-expression of the surface markers CD31, CD133, CD34 and VEGFR2 (13). The characteristics of EPCs delineated in the present study are consistent with the common features of EPCs reported in previous studies. In addition, the co-expression of these markers also characterizes the isolated EPCs at a specific maturation stage (14). EPCs have been reported to ameliorate acute myocardial infarction and diabetic erectile dysfunction by promoting angiogenesis (29-30). However, few data are available regarding the role of EPCs in other diabetic complications, particularly in DCM. In the present study, EPC administration markedly improved cardiac function, while fluorescent-labeled EPCs were identified in cardiac tissues by immunofluorescence analysis, suggesting that EPCs act via a direct transdifferentiation into mature cardiomyocytes. These results corresponded to those drawn by Badorff et al (31), showing that EPCs differentiate into cardiomyocytes when co-cultured with neonatal rat cardiomyocytes. Notably, the discovery rate of EPCs was very low in the cardiac tissues of STZ-induced diabetic rats, which further supports the generally accepted concept that apart from direct cell fusion, EPCs may exert a cardioprotective benefit via their paracrine effects (32).

Increased myocardial interstitial fibrosis is a hallmark pathologic feature of DCM and has been considered to be responsible for the diastolic dysfunction occurring in this condition (33). In the present study, 12-week diabetic condition was found to induce interstitial fibrosis in the left ventricle of STZ rats, as demonstrated by morphologic data and the analysis of type I collagen expression. However, EPC administration significantly reduced left ventricular interstitial fibrosis in diabetic rats. These results are consistent with recent findings showing that EPC administration prevents renal interstitial fibrosis in a murine chronic renal failure model (34), as well as in a mouse model of unilateral ureteral obstruction (35). In addition, Liu et al (36) have demonstrated that transplanted EPCs ameliorate carbon tetrachlorideinduced liver fibrosis in rats. These results, along with results drawn from previous studies, suggest an anti-fibrotic effect of EPCs under different pathologic conditions. Nonetheless, the detailed mechanism involved in the anti-fibrotic effects of EPC transplantation needs to be further elucidated.

Hyperglycemia-induced apoptosis is an early event in the pathophysiology of DCM (37). Caspase-3 is considered a primary executioner of apoptosis. In the present study, caspase-3 was observed to have been activated in STZ-induced diabetic rat hearts. The Bcl-2 family is thought to be the critical factor in the apoptotic signaling pathways, and the relative ratio of anti- and pro-apoptotic proteins is crucial for the determination of cell survival or death (38). In this study, the hearts in the DCM group were found to have exhibited an increased Bax expression and a decreased Bcl-2 expression at the protein level. However, these changes were markedly reversed by EPC administration, suggesting that transplantation of EPCs might have the potential to protect cardiomyocytes from apoptotic death in diabetic conditions. These results are consistent with previous ones demonstrating that several cytokines, released by peripheral blood or bone marrow-derived EPC, are potent inhibitors of apoptosis (39-40). Furthermore, a recent study by Sen et al (41) showed that autologous transplantation of the genetic modification of EPCs also inhibited cardiac apoptosis in a rat model of myocardial infarction. Collectively, results in the current study confirmed previous findings demonstrating that cardiomyocytes apoptosis is associated with the progression of DCM, and that EPC implantation has the potential to prevent cardiomyocytes apoptosis in diabetic conditions.

STZ treatment induces systemic generation of reactive oxygen species (ROS) by activating NADPH oxidase, thus leading to cardiac dysfunction (42). To further investigate the mechanisms by which EPCs exert their cardioprotective effects, the expression of NADPH oxidase subunit p67phox and mitochondrial ROS-eliminating enzyme MnSOD was examined. The results showed that EPC transplantation attenuated the increased p67phox expression and the decreased MnSOD expression that were induced by STZ injection. These findings indicate that EPC transplantation ameliorated diabetic cardiac damage by targeting the redox signaling pathways.

In summary, transplantation of bone-marrow EPCs improved cardiac function, protected cardiomyocytes against apoptosis and reduced the extent of myocardial interstitial fibrosis in a STZ-induced diabetic rat model. Additional experiments showed that this cardioprotective effect may be associated with the antioxidative properties of EPCs. Thus, EPC transplantation may be a novel therapeutic approach for the prevention of diabetic myocardial complications.

\section{Acknowledgements}

This study was supported by a grant from the Heilongjiang Province Science Foundation for Youths (Grant no. QC2010040).

\section{References}

1. Battiprolu PK, Gillette TG, Wang ZV, Lavandero S and Hill JA: Diabetic cardiomyopathy: mechanisms and therapeutic targets. Drug Discov Today Dis Mech 7: e135-e143, 2010.

2. Hayat SA, Patel B, Khattar RS and Malik RA: Diabetic cardiomyopathy: mechanisms, diagnosis and treatment. Clin Sci (Lond) 107: 539-557, 2004.

3. Boudina S and Abel ED: Diabetic cardiomyopathy revisited. Circulation 115: 3213-3223, 2007.

4. Adeghate E: Molecular and cellular basis of the aetiology and management of diabetic cardiomyopathy: a short review. Mol Cell Biochem 261: 187-191, 2004.

5. Aneja A, Tang WH, Bansilal S, Garcia MJ and Farkouh ME: Diabetic cardiomyopathy: insights into pathogenesis, diagnostic challenges, and therapeutic options. Am J Med 121: 748-757, 2008.

6. Asahara T, Murohara T, Sullivan A, et al: Isolation of putative progenitor endothelial cells for angiogenesis. Science 275: 964-967, 1997.

7. Hristov M, Erl W and Weber PC: Endothelial progenitor cells: mobilization, differentiation, and homing. Arterioscler Thromb Vasc Biol 23: 1185-1189, 2003.

8. Krenning G, Dankers PY, Drouven JW, et al: Endothelial progenitor cell dysfunction in patients with progressive chronic kidney disease. Am J Physiol Renal Physiol 296: F1314-F1322, 2009.

9. Chen JZ, Zhang FR, Tao QM, Wang XX and Zhu JH: Number and activity of endothelial progenitor cells from peripheral blood in patients with hypercholesterolaemia. Clin Sci (Lond) 107: 273-280, 2004.

10. Avouac J, Uzan G, Kahan A, Boileau C and Allanore Y: Endothelial progenitor cells and rheumatic disorders. Joint Bone Spine 75: 131-137, 2008. 
11. Muller-Ehmsen J, Braun D, Schneider T, et al: Decreased number of circulating progenitor cells in obesity: beneficial effects of weight reduction. Eur Heart J 29: 1560-1568, 2008.

12. Vasa M, Fichtlscherer S, Aicher A, et al: Number and migratory activity of circulating endothelial progenitor cells inversely correlate with risk factors for coronary artery disease. Circ Res 89: E1-E7, 2001.

13. Allegra A, Coppolino G, Bolignano D, et al: Endothelial progenitor cells: pathogenetic role and therapeutic perspectives. J Nephrol 22: 463-475, 2009.

14. Sen S, McDonald SP, Coates PT and Bonder CS: Endothelial progenitor cells: novel biomarker and promising cell therapy for cardiovascular disease. Clin Sci (Lond) 120: 263-283, 2011.

15. Alev C, Ii M and Asahara T: Endothelial progenitor cells: a novel tool for the therapy of ischemic diseases. Antioxid Redox Signal 15: 949-965, 2011.

16. Grisar JC, Haddad F, Gomari FA and Wu JC: Endothelial progenitor cells in cardiovascular disease and chronic inflammation: from biomarker to therapeutic agent. Biomark Med 5: 731-744, 2011.

17. Georgescu A: Vascular dysfunction in diabetes: The endothelial progenitor cells as new therapeutic strategy. World J Diabetes 2: 92-97, 2011.

18. Kim KA, Shin YJ, Kim JH, et al: Dysfunction of endothelial progenitor cells under diabetic conditions and its underlying mechanisms. Arch Pharm Res 35: 223-234, 2012.

19. Jarajapu YP and Grant MB: The promise of cell-based therapies for diabetic complications: challenges and solutions. Circ Res 106: 854-869, 2010

20. Georgescu A, Alexandru N, Constantinescu A, Titorencu I and Popov D: The promise of EPC-based therapies on vascular dysfunction in diabetes. Eur J Pharmacol 669: 1-6, 2011.

21. Grapensparr L, Olerud J, Vasylovska S and Carlsson PO The therapeutic role of endothelial progenitor cells in Type 1 diabetes mellitus. Regen Med 6: 599-605, 2011.

22. Zhang SJ, Zhang H, Hou M, et al: Is it possible to obtain 'true endothelial progenitor cells' by in vitro culture of bone marrow mononuclear cells? Stem Cells Dev 16: 683-690, 2007.

23. Timmermans F, Plum J, Yoder MC, Ingram DA, Vandekerckhove B and Case J: Endothelial progenitor cells: identity defined? J Cell Mol Med 13: 87-102, 2009.

24. Cheng Y, Liu G, Pan Q, Guo S and Yang X: Elevated expression of liver $\mathrm{X}$ receptor alpha (LXRalpha) in myocardium of streptozotocin-induced diabetic rats. Inflammation 34: 698-706, 2011.

25. Wichi R, Malfitano C, Rosa $\mathrm{K}$, et al: Noninvasive and invasive evaluation of cardiac dysfunction in experimental diabetes in rodents. Cardiovasc Diabetol 6: 14, 2007.

26. Devanesan AJ, Laughlan KA, Girn HR and HomerVanniasinkam S: Endothelial progenitor cells as a therapeutic option in peripheral arterial disease. Eur J Vasc Endovasc Surg 38: 475-481, 2009

27. Prater DN, Case J, Ingram DA and Yoder MC: Working hypothesis to redefine endothelial progenitor cells. Leukemia 21 : $1141-1149,2007$
28. Hirschi KK, Ingram DA and Yoder MC: Assessing identity, phenotype, and fate of endothelial progenitor cells. Arterioscler Thromb Vasc Biol 28: 1584-1595, 2008.

29. Gou X, He WY, Xiao MZ, et al: Transplantation of endothelial progenitor cells transfected with VEGF165 to restore erectile function in diabetic rats. Asian J Androl 13: 332-338, 2011.

30. Park JH, Yoon JY, Ko SM, et al: Endothelial progenitor cell transplantation decreases lymphangiogenesis and adverse myocardial remodeling in a mouse model of acute myocardial infarction. Exp Mol Med 43: 479-485, 2011.

31. Badorff C, Brandes RP, Popp R, et al: Transdifferentiation of blood-derived human adult endothelial progenitor cells into functionally active cardiomyocytes. Circulation 107: 1024-1032, 2003.

32. Zampetaki A, Kirton JP and Xu Q: Vascular repair by endothelial progenitor cells. Cardiovasc Res 78: 413-421, 2008.

33. Asbun J and Villarreal FJ: The pathogenesis of myocardial fibrosis in the setting of diabetic cardiomyopathy. J Am Coll Cardiol 47: 693-700, 2006.

34. Sangidorj O, Yang SH, Jang HR, et al: Bone marrow-derived endothelial progenitor cells confer renal protection in a murine chronic renal failure model. Am J Physiol Renal Physiol 299: F325-F335, 2010.

35. Ma YY, Sun D, Li J and Yin ZC: Transplantation of endothelial progenitor cells alleviates renal interstitial fibrosis in a mouse model of unilateral ureteral obstruction. Life Sci 86: 798-807, 2010.

36. Liu F, Liu ZD, Wu N, et al: Transplanted endothelial progenitor cells ameliorate carbon tetrachloride-induced liver cirrhosis in rats. Liver Transpl 15: 1092-1100, 2009.

37. Cai L, Li W, Wang G, Guo L, Jiang Y and Kang YJ: Hyperglycemia-induced apoptosis in mouse myocardium: mitochondrial cytochrome C-mediated caspase-3 activation pathway. Diabetes 51: 1938-1948, 2002.

38. Burlacu A: Regulation of apoptosis by Bcl-2 family proteins. J Cell Mol Med 7: 249-257, 2003.

39. Kinnaird T, Stabile E, Burnett MS, et al: Marrow-derived stromal cells express genes encoding a broad spectrum of arteriogenic cytokines and promote in vitro and in vivo arteriogenesis through paracrine mechanisms. Circ Res 94: 678-685, 2004.

40. Urbich C, Aicher A, Heeschen C, et al: Soluble factors released by endothelial progenitor cells promote migration of endothelial cells and cardiac resident progenitor cells. J Mol Cell Cardiol 39: 733-742, 2005.

41. Sen S, Merchan J, Dean J, et al: Autologous transplantation of endothelial progenitor cells genetically modified by adenoassociated viral vector delivering insulin-like growth factor-1 gene after myocardial infarction. Hum Gene Ther 21: 1327-1334, 2010.

42. Oelze M, Knorr M, Schuhmacher S, et al: Vascular dysfunction in streptozotocin-induced experimental diabetes strictly depends on insulin deficiency. J Vasc Res 48: 275-284, 2011. 